\title{
Trasplante con precursores hematopoyéticos de cordón umbilical en adultos con neoplasias hematológicas en Chile: Serie clínica
}

\author{
Mauricio Burotto $\mathrm{P}^{1}$, Pablo Bertín C-M ${ }^{1}$, Héctor $\mathbf{G}$ alindo $A^{1}$, \\ Marcelo Garrido $S^{1}$, Geraldine Melgoza $\mathrm{P}^{1}$, Bruno Nervi $\mathbf{N}^{1}$, \\ Pablo Ramírez $V^{1}$, Mauricio 0 cqueteau $T^{1}$, Pelayo Besa de $C^{2}$, \\ Verónica G arcía V 2a, Verónica Jara A 2a, Elvira Álvarez J3a, \\ Maritsa Betancur B ${ }^{3 a}$, Mirtza Rivera ${ }^{3 a}$, Manuel Álvarez $Z^{1}$.

\section{U mbilical cord hematopoietic precursors transplantation. Report of five patients}

Hematopoietic precursors transplantation is a therapeutic alternative for leukemia, some metabolic diseases and some immune deficiency syndromes. In its allogeneic variety, leukemia eradication is based in the conditioning prior to transplantation and the allograft effect against leukemia. Umbilical cord blood is an alternative source of hematopoietic precursors when there are no HLA compatible relatives available. Between 2003 and 2007 we have performed five umbilical cord blood transplant in adult patients in a University hospital. All patients had malignant diseases. Conditioning protocols were ablative in all except in one patient and in all, more than one unit of umbilical cord blood was used. Hematopoietic engraftment was confirmed in all patients and the main complications registered were infectious and associated to immunosuppression (Rev Méd Chile 2009; 137: 1054-60). (Key words: Cord blood stem cell transplantation; Fetal blood; Leukemia)

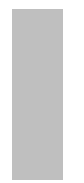

$\mathrm{E}$ trasplante de precursores hematopoyéticos se inició en los años 1970-1979 en el tratamiento de leucemias. En sus fundamentos está el

$\overline{\text { Correspondencia a: Dr. Manuel Álvarez. Departamento de }}$ Hematología-Oncología, Pontificia Universidad Católica de Chile. Diagonal Paraguay 319, Santiago. Fono: 56-2-3546919. Fax: 56-2-2472327. E mail: malvarez@med.puc.cl erradicar la enfermedad por medio de quimioterapia y radioterapia en altas dosis, siendo el injerto o trasplante la plataforma celular de rescate para sostener la hematopoyesis ${ }^{1}$. Esta herramienta terapéutica ha impactado en las posibilidades de curación de muchas enfermedades, siendo la primera elección en leucemias de alto riesgo de recaída, aplasia medular severa, inmunodeficiencias congénitas específicas y recaídas de linfomas ${ }^{2}$. 
Existen dos tipos de trasplante, el autólogo, con células madre del propio paciente y el alogénico con precursores hematopoyéticos genéticamente diferentes. Este último se subdivide en: relacionado, cuando el donante es un familiar y en no relacionado, si no lo es. En este último caso, los precursores proceden de la médula ósea o sangre periférica de donantes voluntarios, o de sangre de cordón umbilical ${ }^{3}$. En sus inicios el éxito del trasplante alogeneico de precursores hematopoyéticos (TAPH) en la curación de leucemias se atribuía a las altas dosis de quimioterapia utilizadas en el condicionamiento; en la actualidad la eficacia del trasplante alogénico, se fundamenta también en el efecto injerto versus leucemia (IVL). El IVL se caracteriza porque los linfocitos $\mathrm{T}$ del donante se encargan de erradicar la enfermedad mediante citotoxicidad contra los blastos leucémicos, efecto gatillado por incompatibilidad antigénica $^{4,5}$. La principal limitante para TAPH es la fuente de células troncales hematopoyéticas, ya que el contar con un donante relacionado compatible se da sólo en 30\% de los casos. Cuando esto no ocurre, el paso siguiente es buscar un donante no relacionado en registros internacionales, proceso prolongado y de costo muy elevado ${ }^{6}$.

La sangre de cordón ha dejado de ser sólo una fuente de precursores hematopoyéticos alternativa, como se utilizó inicialmente en población pediátrica, y es en la actualidad considerada como terapia de primera opción en algunos centros de trasplante en el mundo cuando no existe un familiar compatible ${ }^{7}$. Sus ventajas incluyen no sólo la rapidez en su disponibilidad, sino también beneficios biológicos, como por ejemplo, con menos enfermedad injerto contra huésped (EICH), mayor tolerancia antigénica y lo que es más importante, equivalencia en resultados clínicos respecto a trasplantes alogénicos no relacionados tanto en niños como en adultos ${ }^{8}$. A nivel mundial ha aumentado rápidamente el número de bancos de sangre de cordón los cuales en su mayoría están en Europa y Norteamérica. Actualmente se dispone de más de 300.000 unidades tipificadas y disponibles para ser utilizadas. Pese a la creciente disponibilidad falta aún experiencia en su realización, es en la población pediátrica donde se inició su uso y se han sentado las bases para su utilización en adultos. Las indicaciones para patologías específicas, las mejores combinaciones de drogas y radioterapia para condicionamiento están aún en desarrollo y dependen todavía de la experiencia particular del centro donde se reali$\mathrm{za}^{9}$.

La experiencia en trasplante de cordón en adultos es nueva en Latinoamérica, nuestra intención es posicionar esta modalidad de trasplante como una alternativa factible y segura en nuestro país. Presentamos a continuación los cinco primeros pacientes adultos sometidos a trasplante de cordón en Chile.

\section{Casos clínicos}

Desde diciembre del año 2003 hasta febrero de 2007 se realizaron cinco trasplantes de precursores de cordón umbilical en adultos, los protocolos de condicionamiento, mieloablativo o no, así como el número de unidades a ser infundidas fueron consideradas caso a caso, dependiendo de variables como peso del receptor, número de células CD34 por unidad, tipo de enfermedad que motivó la indicación de trasplante y comorbilidades del paciente.

Paciente 1. Hombre de 54 años, portador de leucemia mieloide aguda M4, diagnosticada en julio de 2003, refractario a terapia de inducción. Por no contar con donante relacionado compatible, se realizó trasplante de cordón en diciembre de 2003 infundiéndose 3 unidades con condicionamiento no mieloablativo (Tablas 1 y 2 ). El prendimiento de neutrófilos se constató el día +30 postrasplante y el de plaquetas el día +55 . En el postrasplante inmediato el paciente presentó cuadro de sepsis por hongos y citomegalovirus que requirió tratamiento antiviral y antifúngico prolongado evolucionando favorablemente. Fue dado de alta hospitalaria al día +42 . Presentó EICH cutánea grado 2 en controles ambulatorios, el que fue manejado con corticoides. La recaída de su leucemia se presentó a los 5 meses y falleció por insuficiencia respiratoria.

Paciente 2. Hombre de 35 años, con diagnóstico de leucemia mieloide crónica en el año 2000, en tratamiento con imatinib con buena respuesta hasta abril de 2004. En agosto de ese año presentó crisis blástica, por lo que recibió 2 ciclos de 
Tabla 1. C aracterísticas de los pacientes, acondicionamiento y unidades de cordón

\begin{tabular}{|c|c|c|c|c|c|c|}
\hline Sexo & E dad & D iagnóstico & Condicionamiento & SCU 1 & SCU 2 & $\begin{array}{c}\text { Dosis } \\
\text { (leucocitos } \times\left(10^{6}\right)\end{array}$ \\
\hline M & 54 & LMA fallo inducción & Flu-Cy- TBI & $4 / 6$ & $5 / 6$ & 50 \\
\hline $\mathrm{M}$ & 35 & LMC fase acelerada & Bu-Cy- ATG & $4 / 6$ & $4 / 6$ & 40 \\
\hline $\mathrm{F}$ & 16 & LMA RC1 & Flu-Cy-TBI & $5 / 6$ & $5 / 6$ & 40 \\
\hline $\mathrm{F}$ & 39 & MDS & Flu-Cy-TBI & $5 / 6$ & $5 / 6$ & 39 \\
\hline M & 26 & LMA RC2 & Flu-Cy-TBI & $5 / 6$ & $6 / 6$ & 40 \\
\hline ATG & \multicolumn{2}{|c|}{ : Globulina antitimocito } & MDS & \multicolumn{3}{|c|}{ : Mielodisplasia } \\
\hline Cy & \multicolumn{2}{|c|}{ : Ciclofosfamida } & $\mathrm{RC}$ & \multicolumn{3}{|c|}{ : Remisión completa } \\
\hline Flu & \multicolumn{2}{|c|}{ : Fludarabina } & SCU & \multicolumn{3}{|c|}{ : Sangre cordón umbilical } \\
\hline LMA & \multicolumn{2}{|c|}{ : Leucemia mieloide aguda } & TBI & \multicolumn{3}{|c|}{ : Total body irradiation } \\
\hline LMC & : Leuc & mia mieloide crónica & & & & \\
\hline
\end{tabular}

Tabla 2. Evolución postrasplante prendimiento, EICH y condición actual

\begin{tabular}{|c|c|c|c|c|c|c|}
\hline Sexo & Edad & $\begin{array}{l}\text { RAN } \\
>500\end{array}$ & $\begin{array}{l}\text { Plts } \\
>20.000\end{array}$ & Q uimerismo & EICH & Resultado \\
\hline M & 54 & +30 & +55 & $100 \%$ SCU 1 & $\begin{array}{l}\text { Agudo } \\
\text { G2 }\end{array}$ & $\begin{array}{l}\text { Recidiva medular } \\
+150\end{array}$ \\
\hline M & 35 & +26 & - & 100\% SCU 1 & No & $\begin{array}{l}\text { Fallece por hemorragia } \\
\text { digestiva }+55\end{array}$ \\
\hline $\mathrm{F}$ & 16 & +17 & +35 & 100\% SCU 2 & $\mathrm{No}$ & $\begin{array}{l}\text { En remisión, sin EICH, } \\
2 \text { años }\end{array}$ \\
\hline $\mathrm{F}$ & 39 & +32 & +67 & 100\% SCU 2 & $\begin{array}{l}\text { Agudo } \\
\text { G3 }\end{array}$ & $\begin{array}{l}\text { Fallece IRA } \\
\text { Shock séptico, }+210\end{array}$ \\
\hline M & 26 & +20 & +44 & 100\% SCU 2 & $\begin{array}{l}\text { Agudo } \\
\text { G3 }\end{array}$ & $\begin{array}{l}\text { En remisión, sin EICH, } \\
1,5 \text { años }\end{array}$ \\
\hline
\end{tabular}

EICH : Enfermedad injerto contra huésped

G : : Grado

IRA : Insuficiencia renal aguda

Plts : : Plaquetas

Quimerismo : Reconstitución de la hematopoyesis en el receptor, en relación al número de unidad de cordón RAN : Recuento absoluto neutrófilos

SCU : Sangre de cordón umbilical

inducción, logrando remisión completa. Se realizó trasplante de cordón en diciembre de 2004, con infusión de 2 unidades usando condicionamiento mieloablativo (Tablas 1 y 2 ). El prendimiento medular se constató el día +26 . Evolucionó con EICH aguda cutánea grado II e intestinal grado III, manejado inicialmente con corticoides, se complicó en su evolución en las semanas siguientes con hemorragia digestiva y sepsis de foco abdominal en el día +55. Falleció en shock séptico refractario. No se alcanzó a evidenciar alza en los recuentos plaquetarios.

Paciente 3. Mujer de 39 años, con diagnóstico mielodisplasia AREB el año 2005, uso de eritropoyetina hasta febrero de 2006. Se constató 
aumento progresivo de blastos hasta $14 \%$ en mayo de 2006. En junio de 2006 se realizó trasplante de cordón con 2 unidades, condicionamiento mieloablativo (Tablas 1 y 2). Prendimiento medular el día +32 y de plaquetas el +67 . Evolucionó con EICH cutánea grado 2 el día +30 e intestinal grado II el día +70 . El día +120 presentó falla renal aguda por ciclosporina, ésta mejoró hasta creatininemia de $1,5 \mathrm{mg} / \mathrm{dl}$ después de la suspensión de la droga. Ingresó día +200 con falla renal aguda, se sospechó microangiopatía trombótica de evolución subaguda, la que no se confirmó, evolucionó con sepsis de foco respiratorio y falleció de falla multiorgánica.

Paciente 4. Mujer de 16 años, con diagnóstico de LMA M6 de alto riesgo, diagnosticada en enero 2006, se realizó trasplante de cordón en junio de 2006 en primera remisión, se infundieron 2 unidades, condicionamiento mieloablativo (Tablas $1 \mathrm{y}$ 2). Evolucionó en el período inmediato postrasplante con hipoparatiroidismo funcional, sin causa demostrada. Con prendimiento medular el día +17 y de plaquetas el día +32 . Alta hospitalaria día +36 . Buena evolución, sin evidencia de recidiva a los dos años de seguimiento.

Paciente 5. Hombre 26 años con diagnóstico de LMA M2 desde octubre 2005, inició tratamiento logrando remisión en mayo 2006. Presentó recaída en octubre del mismo año, se reindujo logrando remisión y se realizó trasplante de cordón en febrero de 2007, se infundieron 2 unidades, condicionamiento ablativo (Tablas $1 \mathrm{y}$ 2). Evolucionó a la cuarta semana postrasplante con EICH aguda cutánea grado III e intestinal grado II, con buena respuesta a terapia esteroidal. Presentó prendimiento medular día +20 y plaquetario día +44 . Evolucionó en este período con infección por citomegalovirus que requirió tratamiento prolongado con ganciclovir. El día +120 evolucionó con sepsis de foco pulmonar en la cual se logró demostrar la presencia de 3 agentes, virus sincicial respiratorio, aspergillus y bocavirus (la búsqueda de este último agente comienza el año 2007 en nuestra institución), evolucionó en forma favorable siendo dado de alta. Actualmente en buenas condiciones sin signos clínicos de EICH crónica y en remisión a los 16 meses de seguimiento.

\section{DisCUSIÓN}

El trasplante de cordón es una alternativa frente a la necesidad de un trasplante alogénico y comparte con éste la propiedad de erradicar la enfermedad por medio del efecto e injerto contra leucemia. Se utiliza como primera opción en centros específicos de Estados Unidos de Norteamérica y Japón y no se conocen aún experiencias en Chile con esta modalidad de trasplante en pacientes adultos ${ }^{7}$.

En los resultados observados en esta serie destaca el grupo variado de patologías que motivó la necesidad de un TAPH. Considerando que mundialmente la experiencia en trasplante de cordón está en desarrollo, la selección del candidato es un elemento aún en discusión. El primero de nuestros pacientes en términos pronósticos no sólo sumaba una patología de alto riesgo sino también la edad límite para un TAPH, razón por la cual se eligió un condicionamiento no ablativo. Estos esquemas se han utilizado más en los últimos 10 años y se basan en el uso de quimioterapia y radioterapia en dosis más bajas. Esto permite mielosuprimir transitoriamente y disminuir al máximo la toxicidad no hematológica (gastrointestinal, renal y hepática), con intención de trasplantar pacientes mayores o con más comorbilidades, manteniendo el efecto ICL característico del TAPH ${ }^{10-12}$.

Otra característica de nuestra serie fue el utilizar dobles y triples unidades de sangre de cordón (SCU), como estrategia la primera es ampliamente utilizada en algunos centros y tiene como fundamento el alcanzar el número crítico de precursores de acuerdo al peso del receptor ${ }^{13-15}$. Laughlin y cols, en 54 pacientes adultos, demostraron la importancia de la dosis de células en relación al prendimiento medular, demostrándose una ventaja significativa del uso de $2,5 \times 10^{7} / \mathrm{kg}$ células nucleadas infundidas o mayor ${ }^{16}$. Algunas experiencias retrospectivas recientemente publicadas comparan el uso de dobles SCU con una unidad, mostrando menos recaída de la enfermedad y una tendencia a la mayor sobrevida, al costo de una mayor EICH aguda, esto último probablemente reflejando mayor efecto injerto contra el tumor ${ }^{17}$. En relación a los resultados nuestra serie muestra en todos los casos prendimiento hematopoyético, sólo un paciente no presentó alza plaquetaria al momento de su muerte. Fallecieron tres pacientes 
antes del día 100 postrasplante, siendo la morbimortalidad relacionada fundamentalmente a complicaciones infecciosas en el contexto de inmunosupresión y sólo uno a recaída de la enfermedad de base. Dos pacientes se encuentran vivos y en remisión hasta el momento del presente reporte.

Las series internacionales de trasplante de cordón en adultos muestran cifras favorables en resultados y equivalencia con el trasplante convencional. La experiencia europea publicada por Rocha y cols (EUROCORD), en pacientes con leucemia aguda, compara los resultados de trasplante SCU en 98 pacientes con 584 que recibieron un trasplante de médula ósea de donante no relacionado (TMNR), la mortalidad relacionada al trasplante (MRT) $(44 \%$ y $38 \%$ P $=0,13)$ y la sobrevida libre de enfermedad (SLE) (36\% y 42\% P $=0,08$ ) fue igual en ambos grupos, mientras que la incidencia de EICH aguda fue menor (26\% y 39\% $\mathrm{P}=0,008)^{18}$. La experiencia americana publicada por Laughlin y cols, compara los resultados de 150 pacientes sometidos a trasplante de SCU, con 450 pacientes con TMNR. El tiempo de recuperación medular fue mayor en el grupo de SCU (27 y 18 días $\mathrm{P}<0,001$ ), pero la SLE fue similar en los dos grupos $^{19}$. Este retardo en el prendimiento medular se observó también en nuestra serie, siendo el promedio 25 días para los leucocitos y 50 días para plaquetas, cifras que se comparan a lo descrito en las grandes series. Se observó quimerismo en $100 \%$ de los casos predominando siempre una de las dos unidades de cordón infundidas (Tabla 2).

La serie japonesa de Takahashi y cols, compara los resultados en 100 pacientes sometidos a trasplante SCU con pacientes con trasplante relacionado de precursores de sangre periférica, demostrando similar resultado en MRT (9\% y 13\%) y
SLE $(70 \% \text { y } 60 \%)^{20}$. Todas estas series son retrospectivas y existe en mayor o menor grado el sesgo en la selección de los pacientes para el trasplante. La Tabla 3 resume las características principales de las series internacionales comentadas anteriormente. El número de complicaciones infecciosas que se presentan en este tipo de trasplante así como el tipo inhabitual de agentes oportunistas, están descritas en trasplantes de SCU. Esto se explica por el prendimiento medular tardío que expone a tiempos de neutropenia prolongados y al uso de agentes altamente inmunosupresores como la fludarabina para la prevención de $\mathrm{EICH}^{21,22}$. La morbimortalidad infecciosa y presencia de agentes oportunistas como citomegalovirus y aspergillus está presente en nuestra serie y se asocian en 2 casos por lo menos a injuria inflamatoria producto de EICH. Es por esta misma asociación que es necesario plantear nuevas estrategias que permitan un control equilibrado del rechazo y EICH, condicionamiento y manejo inmunosupresor postrasplante y al mismo tiempo disminuir la incidencia de infecciones oportunistas, mejorando variables como la temporalidad y la magnitud de las profilaxis infecciosas que se deben utilizar en relación a esta modalidad de trasplante.

Estos son los primeros cinco casos en adultos comunicados en nuestro país, la importancia de esta experiencia radica en las proyecciones que puede tener esta terapia para países como Chile, donde la factibilidad de trasplantes en base a donantes no es viable a gran escala. Este esfuerzo a nivel local combinado con el seguimiento a más de 10 años de series internacionales en adultos, pueden dar paso a una era en la cual la fuente de precursores hematopoyéticos no será un obstáculo para el tratamiento con intención curativa de un gran número de pacientes. 
Tabla 3. Características TAPH con células de cordón en adultos. Series internacionales

\begin{tabular}{|c|c|c|c|c|}
\hline & $\begin{array}{l}\text { Barker } \\
2003\end{array}$ & $\begin{array}{l}\text { Rocha } \\
2004\end{array}$ & $\begin{array}{l}\text { Laughlin } \\
2004\end{array}$ & $\begin{array}{l}\text { Takahashi } \\
2007\end{array}$ \\
\hline Número pctes y & 43 pctes & 98 pctes & 150 pctes & 100 pctes \\
\hline tipo de reporte & $\begin{array}{l}18 \text { LMA } \\
\text { retrospectivo }\end{array}$ & $\begin{array}{c}\text { LMA } \\
\text { retrospectivo }\end{array}$ & $\begin{array}{c}\text { LMA } \\
\text { retrospectivo }\end{array}$ & $\begin{array}{l}57 \text { LMA } \\
\text { prospectivo }\end{array}$ \\
\hline Edad & 49 & 24,5 & $\begin{array}{l}48 \%>30 a \\
26 \%>40 a\end{array}$ & 38 \\
\hline Peso prom & 73 & 58 & 68 & 55 \\
\hline Match HLA, & $20(51 \%)$ & 5/6:6 pctes & $6 / 6: 0$ pctes & $6 / 6: 0$ \\
\hline donante-receptor & 1-2 mismatch & 4/6:48 pctes & 5/6:34 pctes & $5 / 6: 16$ \\
\hline & & $\begin{array}{l}\text { 3/6:37 pctes } \\
\text { 2/6:4 pctes }\end{array}$ & 4/6:116 pctes & $\begin{array}{c}4 / 6: 54 \\
3 / 6: 28 \\
2 / 6: 2\end{array}$ \\
\hline $\begin{array}{l}\text { Células nucleadas } \\
\text { infundidas }\end{array}$ & $3,2 \times 10^{7} / \mathrm{kg}$ & $2,3 \times 10^{7} / \mathrm{kg}$ & $2,2 \times 10^{7} / \mathrm{kg}$ & $2,43 \times 10^{7} / \mathrm{kg}$ \\
\hline Prendimiento & 5-28 días & 14-80 días & 25-29 días & 16-46 días \\
\hline mieloide & prom: 9,5 d & prom: $26 \mathrm{~d}$ & prom: $27 \mathrm{~d}$ & prom: $22 \mathrm{~d}$ \\
\hline EICH aguda & II-IV:44\% & II-IV:26\% & II-IV:40\% & II-IV:51\% \\
\hline Grado & III-IV:9\% & III-IV:13\% & III-IV:NR & III-IV:6\% \\
\hline Falla prendimiento & 1 pcte & $\begin{array}{l}20 \text { pctes } \\
(20 \%)\end{array}$ & NR & $\begin{array}{l}9 \text { pctes } \\
(9 \%)\end{array}$ \\
\hline
\end{tabular}

EICH : Enfermedad injerto contra huésped

LMA : Leucemia mieloide aguda

Match HLA : Compatibilidad complejo mayor de histocompatibilidad

NR : No reportado

Pcte : Paciente

Prom : Promedio

TAPH : Trasplante alogénico de precursores hematopoyéticos

\section{REFERENCIAS}

1. Copelan EA. Hematopoietic stem-cell transplantation. N Engl J Med 2006; 354: 1813-26.

2. International Bone Marrow Transplant Registre (IBMTR). State of the art in clinical transplantation 2003. Autologous Blood and Marrow Transplant Registry (ABMTR).

3. Grewal SS, Barker JN, Davies SM, Wagner JE. Unrelated donor hematopoietic cell transplantation: marrow or umbilical cord blood? Blood 2003; 101: 4233-44.

4. Champlin R, Khouri I, Shimoni A, Gajewski J, Kornblau S, Molldrem J ET AL. Harnessing graft-versus-malignancy: non-myeloablative preparative regimens for allogeneic haematopoietic transplantation, an evol- ving strategy for adoptive immunotherapy. $\mathrm{Br} \mathrm{J}$ Haematol 2000; 111: 18-29.

5. Baron F, SAndmaier MB. Chimerism and outcomes after allogeneic hematopoietic cell transplantation following nonmyeloablative conditioning. Leukemia 2006; 20: 1690-700.

6. Arcese W, Rocha V, Labopin M, Sanz G, Iori AP, De LimA M ET AL. Unrelated cord blood transplants in adults with hematologic malignancies. Haematologica 2006; 91: 223-30.

7. Barker JN, Rocha V, Scaradavou A. Optimizing unrelated donor cord blood transplantation. Biol Blood Marrow Transplant 2008; 15 (1 Suppl): 154-61.

8. Ooi J, Iseki T, Takahashi S, Tomonari A, Takasugi K, ShimohaKamada Y ET al. Unrelated cord blood trans- 
plantation for adult patients with de novo acute myeloid leukemia. Blood 2004; 103: 489-91.

9. Schoemans H, Theunissen K, Maertens J, Boogaerts M, Verfaillie C, Wagner J. Adult umbilical cord blood trasplantation: a comprehensive review. Bone Marrow Transplantation 2006; 38: 83-93.

10. Baron F, Sandmaier BM. Current status of hematopoietic stem cell transplantation after nonmyeloablative conditioning. Curr Opin Hematol 2005; 12: 435-43.

11. Baron F, Baker JE, Storb R, Gooley TA, Sandmaier BM, MARIS MB ET AL. Kinetics of engraftment in patients with hematologic malignancies given allogeneic hematopoietic cell transplantation after nonmyeloablative conditioning. Blood 2004; 104: 2254-62.

12. Chao NJ, Кoh LP, Long GD, Gasparetto C, Horwitz M, Morris A ET AL. Adult recipients of umbilical cord blood transplants after nonmyeloablative preparative regimens. Biol Blood Marrow Transplant 2004; 10: $569-75$.

13. Haspel RL, Ballen KK. Double cord blood transplants: filling a niche? Stem Cell Rev 2006; 2: 81-6.

14. Ballen KK, Spitzer TR, Yeap BY, Mcafee S, Dey BR, AtTAR E ET AL. Double unrelated reduced-intensity umbilical cord blood transplantation in adults. Biol Blood Marrow Transplant 2007; 13: 82-9.

15. De Lima M, St John LS, Wieder ED, Lee MS, Mcmannis J, Karandish S ET AL. Double-chimaerism after transplantation of two human leucocyte antigen mismatched, unrelated cord blood units. Br J Haematol 2002; 119: 773-6.

16. Laughlin MJ, Barker J, Bambach B, Koc ON, Rizzieri DA, WAGNER JE ET AL. Hematopoietic engraftment and survival in adult recipients of umbilical-cord blood from unrelated donors. N Engl J Med 2001; 344: 1815-22.
17. Veneris MR, Brunstein C, Deford RE, Barker J, Weisdorf DJ, Blazar BR et al. Risk of relapse after umbilical blood cord transplantation in patients with acute leukemia: marker reduction in recipients of two units. ASH Annual Meeting Abstracts 2005; 106: 93a. Abstract 305.

18. Rocha V, Labopin M, Sanz G, Arcese W, Schwerdtfeger R, Bosi A ET AL. Transplants of umbilical-cord blood or bone marrow from unrelated donors in adults with acute leukemia. N Engl J Med 2004; 351: 227685.

19. Laughin MJ, Eapen M, Rubinstein P, Wagner JE, Zhang MJ, Champlin RE et al. Outcomes after transplantation of cord blood or bone marrow from unrelated donors in adults with leukemia. N Engl J Med 2004; 351: 2265-75.

20. Takahashi S, Ooi J, Tomonari A, Konuma T, Tsukada N, Oiwa-Monna M ET al. Comparative single-institute analysis of cord blood transplantation from unrelated donors with bone marrow or peripheral blood stem cell transplantation from related donors in adult patients with hematologic malignancies after myeloablative conditioning regimen. Blood 2007; 109: 1322-30.

21. Tomonari A, Iseki T, Ooi J, Takahashi S, Shindo M, Ishit K ET AL. Cytomegalovirus infection following unrelated cord blood transplantation for adult patients: a single institute experience in Japan. Br J Haematol 2003; 121: 304-11.

22. Tomonari A, Iseki T, Takahashi $S$, Ooi J, Takasugi $K$, SHimohakamada Y ET aL. Varicella-zoster virus infection in adult patients after unrelated cord blood transplantation: a single institute experience in Japan. Br J Haematol 2003; 122: 802-5. 